\title{
Evolución de las maquiladoras en México. Política industrial y aprendizaje tecnológico
}

\author{
Óscar F. Contreras* \\ Luis Felipe Munguía**
}

Resumen: La expansión de las maquiladoras ha generado una parte sustancial del crecimiento industrial en el norte de México, durante los últimos 25 años. Inicialmente, estas plantas se dedicaban a actividades intensivas en mano de obra, operaban con tecnologías rudimentarias y en condiciones precarias; sin embargo, desde mediados de los años ochenta, muchas introdujeron tecnologías de punta, una organización moderna y fuerza de trabajo bien capacitada; asimismo, se delegaron más funciones estratégicas a las plantas locales. Este artículo resume los hallazgos principales de investigaciones recientes en torno a la evolución y consecuencias del modelo de industrialización basado en las maquiladoras, con énfasis en el proceso de aprendizaje tecnológico asociado a la trayectoria industrial de la región. Se concluye que la estrategia de industrialización basada en el costo salarial está agotada. Ahora, la opción es transitar hacia la especialización en segmentos industriales apoyados en el conocimiento y de alto valor agregado.

Palabras clave: maquiladoras, aprendizaje tecnológico, economía del conocimiento.

Abstract: Expansion of the maquiladora industry has generated a substantial part of the industrial growth in northern Mexico during the last 25 years. Initially, these plants carried out labor-intensive activities using rudimentary technology under precarious conditions. However, since the mid-eighties, many plants introduced state-of-the-art technologies, modern organization and a well-trained workforce; also, more strategic functions were delegated to local plants. This article summarizes the main findings of recent studies concerning the evolution and conse-

\footnotetext{
* Profesor-investigador del Programa de Relaciones Industriales de El Colegio de Sonora. Correspondencia: Obregón 54, colonia Centro. C. P. 83000. Hermosillo, Sonora, México. Teléfono 01(662) 259-53-00. Correo electrónico: ocontre@colson.edu.mx

** Estudiante de la maestría en Economía, El Colegio de México.
} 
quences of the maquiladora-based model of industrialization, emphasizing the technological learning process associated with the industrial trajectory of the region. The conclusion is that the industrialization strategy based on labor costs has been exhausted. Today, the option is to move toward specialization in knowledge-supported and valueadded industrial segments.

Key words: maquiladoras, technological learning, knowledge economy.

\section{Introducción}

La "industria maquiladora de exportación"1 es uno de los fenómenos más estudiados y debatidos de la realidad social y económica del norte de México. Esta prominencia se debe, en parte, al peso cada vez mayor de tales empresas en los indicadores industriales del país, pero también a la carga política e ideológica que suele estar asociada a su evaluación; es decir, el análisis del desempeño de las maquiladoras por lo general supone una evaluación del modelo de desarrollo industrial del país.

La expansión de las maquiladoras ha generado una parte sustancial del crecimiento industrial en el norte de México, durante los últimos 25 años. Originalmente, estas operaciones industriales formaron parte de una estrategia temporal del gobierno mexicano, para abatir el desempleo en la zona fronteriza con Estados Unidos; sin embargo, al paso del tiempo se convirtieron en una pieza fundamental de la política industrial, y adquirieron una importancia enorme para la economía mexicana. Para las empresas extranjeras, que establecieron operaciones de maquila en México, este esquema resultó muy ventajoso, ya que les permitió reducir drásticamente sus costos laborales, al instalarse en una región geográfica cercana y pagar salarios bajos.

Al inicio, estas plantas se dedicaban a actividades intensivas en mano de obra, operaban con tecnologías rudimentarias y en condiciones precarias; en la actualidad muchas ya cuentan con tecnologías de punta, organización moderna y fuerza de trabajo bien capacitada, que incluye técnicos e ingenieros (Carrillo 2004; Contreras y Hualde 2004). A fines de 2000, tanto la cantidad de establecimientos

\footnotetext{
${ }^{1}$ En una definición general, las maquiladoras son plantas que importan materias primas, componentes y maquinaria, para procesarlos o ensamblarlos en México y después reexportarlos, principalmente a Estados Unidos, pagando impuestos sólo sobre el valor agregado. La definición adoptada por el Instituto Nacional de Estadística, Geografía e Informática (INEGI) indica que “[...] se considera como establecimiento maquilador a aquella unidad económica que realiza una parte del proceso de producción final de un artículo, por lo regular de ensamblado, misma que se encuentra dentro del territorio nacional y mediante un contrato de maquila se compromete con una empresa matriz, ubicada en el extranjero, a realizar un proceso industrial o de servicio destinado a transformar, elaborar o reparar mercancías de procedencia extranjera, para lo cual importa temporalmente partes, piezas y componentes, mismos que una vez terminados son exportados" (INEGI, citado por el Centro de Estudios de las Finanzas Públicas de la Cámara de Diputados 2003).
} 
como de trabajadores llegaron a su punto más alto, y en 2001 inició un periodo de contracción, en gran medida ocasionado por la recesión de la economía estadounidense. En el primer trimestre de 2002, el empleo había disminuido en 22 por ciento y el número de plantas en 20, según la General Accounting Office (GAO 2003). Conforme la economía de Estados Unidos empezó a mostrar signos de mejoría, la mayor parte de esos empleos se recuperaron. Sin embargo, hay evidencias de que la declinación iniciada en 2001 no fue sólo un problema coyuntural, pues México enfrenta una fuerte competencia de China, Centroamérica y el Caribe (GAO 2003; Dussel 2004). Numerosos analistas señalan que la estrategia de industrialización basada en las maquiladoras está agotada, tanto en el modelo de competitividad industrial, centrado en los costos laborales (Capdeville 2005), como en el de un régimen exclusivo de ensamble para la exportación (Villarreal 2003). Aparentemente, la opción es el tránsito hacia un esquema más avanzado de especialización en los segmentos industriales, apoyados en más conocimiento y de mayor valor agregado; de hecho, en la actualidad hay diversos actores empresariales e institucionales involucrados en esfuerzos dirigidos a este tipo de transformación (Moloman 2006).

En este artículo se presenta una visión panorámica de la evolución de las maquiladoras en México, enfatizando tres aspectos: a) los cambios de la política industrial del país respecto de las maquiladoras; b) el efecto del Tratado de Libre Comercio de América del Norte (TLCAN) en su crecimiento y desempeño y c) el aprendizaje tecnológico y la creación de capacidades locales. El texto está dividido en cinco secciones; en la primera parte se describe de manera general el programa de maquiladoras y la evolución de sus indicadores principales; en la segunda se abordan los cambios en la normatividad, tratando de identificar el papel asignado por el gobierno mexicano a estas empresas en el desarrollo industrial del país; en la tercera se revisan algunas de las implicaciones del TLCAN en el crecimiento de las maquiladoras y en la cuarta, que constituye el apartado central de esta trabajo, se trata el aprendizaje industrial y la creación de capacidades institucionales propiciadas por las maquiladoras en el norte de México. Por último, en la quinta, se proponen algunos elementos para un pronóstico sobre el futuro de la maquila en el país.

\section{La importancia de las maquiladoras en la economía mexicana}

Las maquiladoras no constituyen el único programa gubernamental destinado a promover las exportaciones. Entre los más importantes, cabe destacar el Programa de Importación Temporal para la Exportación (PITEX), establecido en 1990, mediante el cual las empresas pueden importar componentes libres de impuestos, siempre y cuando exporten al menos 30 por ciento de sus ventas; a lo largo de la década de los noventa, el PITEX cobró una importancia cada vez mayor, sobre todo para empresas localizadas en regiones no fronterizas.

A pesar de que en los años recientes el gobierno mexicano ha creado diversos programas para la promoción de las exportaciones, el de las maquiladoras sigue 
siendo el más importante. De acuerdo con los datos del anexo estadístico del sexto informe de gobierno de septiembre de 2006 (Gobierno de los Estados Unidos Mexicanos. Presidencia de la república 2006), el valor de las exportaciones de las maquiladoras en 2005 ascendió a más de 97 mil millones de dólares, lo que representó 45.5 por ciento de las exportaciones totales y 55.6 de las manufactureras. Asimismo, había 1166250 personas ocupadas, es decir, las fábricas generaban 37.8 por ciento del empleo.

Como se observa en la gráfica siguiente (que incluye sólo a las empresas oficialmente registradas como maquiladoras), entre 1975 y 2000 el número de plantas se multiplicó por ocho y el personal ocupado por 18.

\section{Gráfica 1}

México: número de plantas y personal ocupado en las maquiladoras

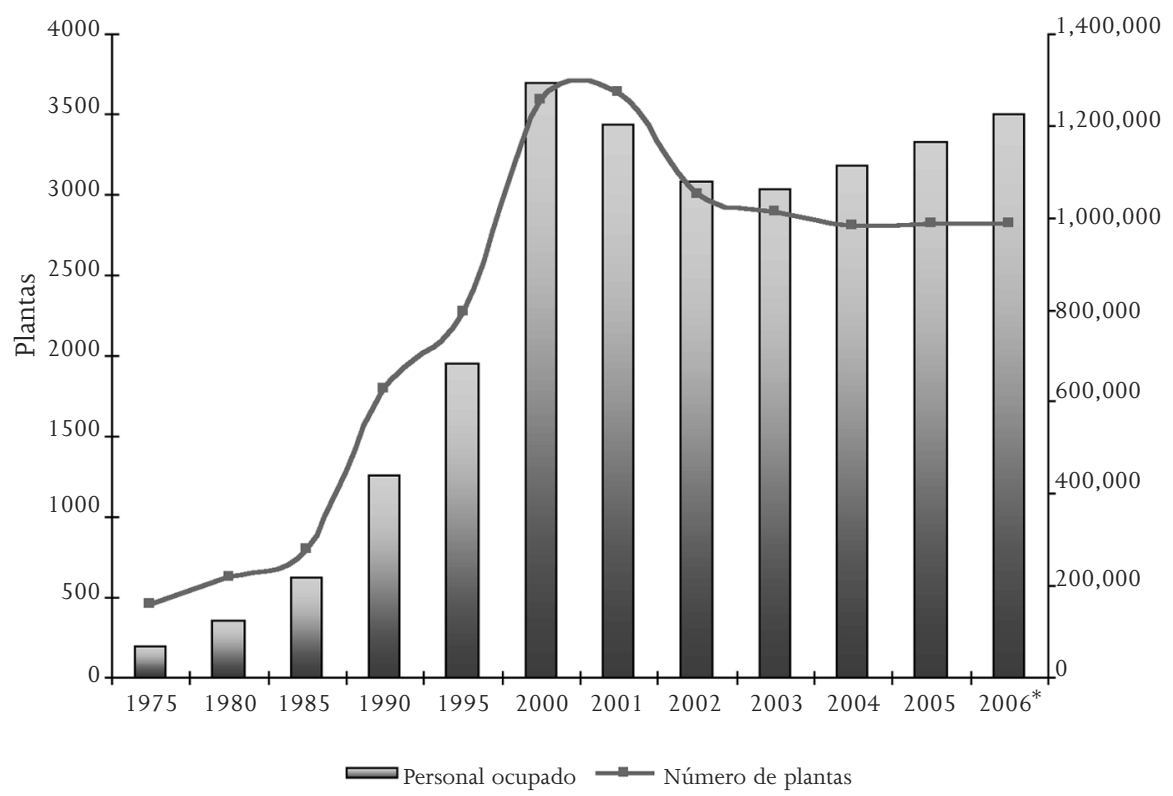

* Promedio anual, excepto para 2006 con datos del mes de junio.

Fuente: elaboración propia, con base en INEGI http://www.inegi.gob.mx/inegi/

El periodo de mayor crecimiento abarca de 1985 a 2000. No sólo se trata del lapso en el que las cifras de producción y empleo alcanzaron sus registros mayores, sino también de una etapa en la que el concepto original de "industria maquiladora” se transformó de manera sustantiva, tanto en los procesos productivos como en la organización industrial y en el marco legal. A finales del 2000 había 3 
703 plantas, con poco más de 1300000 trabajadores. Durante esos años se convirtieron en la fuente principal de empleo industrial y la segunda en generación de divisas; llegaron a representar la mitad de las exportaciones mexicanas y 40 por ciento del empleo manufacturero.

Este crecimiento resulta relevante, porque se trata de un periodo de estancamiento del empleo manufacturero en México. De 1985 a 2000, la tasa de crecimiento anual del trabajo en las maquiladoras fue de 13 por ciento, en tanto que en el resto del sector manufacturero fue menor a 1. En 1985, el total de las exportaciones mexicanas ascendía a poco más de 27 mil millones de dólares, de los cuales 19 por ciento correspondía a las maquiladoras. Para el 2000, el valor total de las exportaciones del país se había multiplicado por seis, y la participación de las maquiladoras representaba ya 48 por ciento.

Las actividades que crecieron más rápido fueron la fabricación de autopartes y la confección, aunque la industria electrónica se mantuvo como la mayor concentradora de plantas y empleados. En el periodo mencionado, la tasa anual del empleo en la fabricación de autopartes fue superior a 20 por ciento, en tanto que en la confección el incremento fue de 14. En la rama electrónica, la tasa de crecimiento fue cercana a 10 por ciento por año. En cuanto a su distribución regional, y a pesar de una tendencia a abrir cada vez más plantas en el resto del país, en la actualidad 77 por ciento de los establecimientos y 83 de empleo continúan localizados en los seis estados fronterizos mexicanos.

Después de crecer casi sin interrupción desde su origen, el número de maquiladoras empezó a declinar de manera acelerada a finales de 2000. En octubre de ese año el empleo llegó a su punto más alto, con 1338970 trabajadores. Pero de noviembre de 2000 a diciembre de 2001 se perdieron poco más de 250 mil puestos de trabajo. A mediados de 2002, el empleo se había reducido en 20 por ciento y la producción en 30. El sector más afectado fue el de la electrónica, especialmente en la región fronteriza (GAO 2003). Sin embargo, cuando la economía de Estados Unidos empezó a recuperarse esa tendencia se revirtió en parte, y al final del primer semestre de 2006 se tenían registradas un total de 2822 plantas y 1223180 empleos (INEGI 2006). Este número de plantas representa apenas 76 por ciento de las que operaban en 2000, pero el empleo había llegado ya a 91 del registrado en aquel año.

Así, a pesar de la crisis severa de 2000 a 2003, la notable recuperación en los años posteriores indica que las maquiladoras siguen teniendo un papel crucial en la economía mexicana, y continúan siendo una opción rentable para las transnacionales de la electrónica, las autopartes y el vestido, entre otras actividades altamente globalizadas. Pero más allá de sus crisis coyunturales y las oscilaciones de sus indicadores principales, es un hecho que se han transformado de manera fundamental. Por una parte, los cambios profundos en la organización productiva han rebasado con mucho el esquema original de ensamblaje de bajo valor agregado para la exportación. Por otro lado, las maquiladoras están destinadas a diluirse paulatinamente, conforme los diferentes programas exportadores homogenicen sus reglas de operación y desaparezca su excepcionalidad como régimen de exportación. 


\section{Maquiladoras y política industrial}

Originalmente, el programa de maquiladoras fue un instrumento para crear empleos. ${ }^{2}$ El gobierno mexicano buscaba enfrentar una situación de desempleo grave en la zona fronteriza con una política de excepción, con alcances temporales y regionales delimitados. En cuanto a su temporalidad, se pretendía que en el mediano plazo las plantas de ensamble crearan eslabonamientos productivos, y promovieran una industrialización de base nacional; en cuanto a su alcance regional, el modelo respondía de forma exclusiva a las condiciones de la frontera norte, pues en el resto del país la política industrial continuaba centrada en la protección de la industria mexicana, mediante instrumentos fiscales y arancelarios.

Entre las consecuencias del hecho de que el programa de maquiladoras estuviera dirigido a una región específica, pero sobre todo el de tratarse de una política en franco antagonismo con el pensamiento proteccionista dominante en el gobierno federal mexicano, es que en realidad no se diseñara ningún instrumento concreto para promover el objetivo explícito de crear eslabonamientos productivos a través de ellas. En el discurso oficial, las maquiladoras nunca dejaron de considerarse un "mal temporal"; en los hechos, el enfoque gubernamental se limitó a permitir su instalación para combatir el desempleo y generar divisas. Según tal enfoque, se elaboraron todos los instrumentos que regularon sus actividades durante los primeros 20 años de operación: en 1966 se emitieron las reglas que permitieron la importación temporal de maquinaria e insumos a la franja fronteriza; en 1971 un reglamento nuevo autorizó el establecimiento de plantas en regiones no fronterizas, y la creación de empresas de capital extranjero en su totalidad y en 1977 se definieron los mecanismos de coordinación, para el cumplimiento de las normas aplicables a este tipo de empresas. En todo ese periodo, los cambios legales fueron precisando las normas administrativas y los mecanismos de operación, pero siempre considerando a las maquiladoras como industrias transitorias, en una situación de excepción (Contreras 2000).

El primer cambio importante sucedió en 1983, en el contexto de lo que sería el principio de un proceso amplio de apertura externa, y desregulación de la economía mexicana. Tanto el Plan Nacional de Desarrollo publicado ese año, como el nuevo Decreto para el fomento y operación de industria maquiladora de exportación, de agosto de 1983, coincidían en otorgar a las maquiladoras un papel relevante no sólo en la generación de empleo y divisas, sino como la base del desarrollo

\footnotetext{
2 La industria maquiladora surgió en 1965, como una combinación de dos instrumentos gubernamentales: las tarifas arancelarias 806.30 y 807.00 en Estados Unidos (después sistema armonizado HTS 9802), y el Programa de Industrialización Fronteriza en México. Para las empresas de Estados Unidos, este esquema resultó muy atractivo ya que les permitió mantener su competitividad en el mercado global, con costos de producción sustancialmente más bajos, al trasladar a México parte del proceso productivo. Tal segmentación fue posible gracias a los avances en las comunicaciones y el transporte, que permitieron mantener una coordinación eficiente y supervisión estrecha de los procesos productivos geográficamente dispersos. Para México, el objetivo principal era atraer empresas extranjeras a la frontera, que emplearan a los trabajadores forzados a regresar al país, al concluir el Programa Bracero en Estados Unidos.
} 
económico de la región fronteriza y fuente de modernización tecnológica (Carrillo 1989). Este decreto les concedió, por primera vez, el estatus de sociedades mercantiles mexicanas, a la vez les permitió vender hasta 20 por ciento de su producción en territorio mexicano; desde entonces los documentos oficiales las empezaron a considerar como un factor de desarrollo industrial y no sólo como enclaves de ensamblaje para crear empleos. Este enfoque se afianzó y precisó en el decreto de 1989, cuando se consideraron como instrumentos de trasferencia de tecnología y promotoras de la competitividad de la industria nacional (Comisión Económica para América Latina y el Caribe 1996). Después, el decreto fue reformado en 1994, para ajustarse a las disposiciones del TLC, y en 1996 se introdujeron cambios para estimular el desarrollo de empresas mexicanas proveedoras de insumos y partes.

El decreto vigente, emitido en 1998 (Diario Oficial de la Federación 1 de junio), resume con claridad la evolución del enfoque gubernamental al establecer que:

Las empresas maquiladoras de exportación deberán atender a las siguientes prioridades nacionales:

I. Crear fuentes de empleo;

II. Fortalecer la balanza comercial del país a través de una mayor aportación neta de divisas;

III. Contribuir a una mayor integración interindustrial y coadyuvar a elevar la competitividad internacional de la industria nacional, y

IV. Elevar la capacitación de los trabajadores e impulsar el desarrollo y la transferencia de tecnología en el país.

Además de formalizar este enfoque y establecer sus prioridades, el decreto de 1998 le quitó la facultad a la entonces Secretaría de Comercio y Fomento Industrial de negar autorización o cancelar permisos por "afectación de la industria nacional no maquiladora”, y definió más claramente las modalidades, incluso la de "empresa maquiladora por servicios".

El "modelo maquilador", diseñado como un recurso de emergencia, se fue transformando así tanto en su normatividad como en su funcionamiento, hasta convertirse en la base del desarrollo industrial en la región norte de México, y en el frente más dinámico de las exportaciones del país. Todos estos cambios legales estuvieron en su momento ligados con los estructurales experimentados por las maquiladoras, y con el reconocimiento de la importancia creciente de estas empresas para la economía mexicana. Como parte de esa evolución continua de las normas en el medio empresarial y gubernamental, se espera la emisión de un decreto nuevo para finales de 2006, y que homologará las reglas de operación para las maquiladoras y las empresas PITEX, con lo que se eliminaría el carácter de excepcionalidad de las maquiladoras, pero se preservarían las ventajas arancelarias y fiscales para las empresas en esquemas de "producción compartida” y de manufactura para la exportación.

Como se puede apreciar en este recuento breve, las transformaciones del marco legal de las maquiladoras no han formado parte de una visión estratégica del desa- 
rrollo industrial y económico, dentro de la cual los instrumentos normativos estuviesen diseñados para aprovechar su expansión y orientar el desarrollo industrial en determinado sentido. Los cambios han consistido principalmente en adaptaciones y ajustes, para adecuar la norma a la realidad eminente del sector y a las demandas empresariales.

\section{El efecto del TLCAN}

Durante los primeros años del TLCAN las maquiladoras crecieron notablemente, a tal grado que para algunos analistas la explicación de tal expansión son las condiciones creadas por el tratado (Vargas 2003). En efecto, en los primeros seis años del TLCAN el empleo creció en 110 por ciento, mientras que en los seis previos había sido de 78 (Sargent y Matthews 2003). Asimismo, durante el mismo lapso el número de plantas creció 49 por ciento, y el incremento fue de 78 entre 1994 y 2000. A pesar de esta aparente correlación positiva, analistas como Gruben y Kiser (2001) han mostrado de manera convincente que este efecto no se puede atribuir de manera directa al tratado. De acuerdo con estos autores, influyen más factores convencionales como el crecimiento de la producción industrial de Estados Unidos y el diferencial del salario manufacturero mexicano frente al del vecino del norte y de los países asiáticos (Gruben y Kiser 2001, 23). ${ }^{3}$

Entonces, más que hablar de un "efecto del TLCAN", para explicar el crecimiento sin precedentes de las maquiladoras, habría que analizar sus particularidades "en el contexto del TLCAN". En este sentido, hay factores dignos de mención como las normas regionales, que penalizan el uso de insumos procedentes de países ajenos a la región, representaron un incentivo para la fabricación de partes y componentes en México. Además, con la eliminación de barreras al comercio y la inversión, el tratado fortaleció las estrategias de organización productiva que involucran al espacio geográfico del TLCAN, como una región económica integrada. Estas estrategias son evidentes sobre todo en las industrias automotriz y electrónica, pero se advierten también en otras como el equipo de cómputo y la confección de ropa. Las empresas transnacionales racionalizan la producción sobre bases regionales o globales, y si bien los costos salariales son un factor clave en todas las decisiones de inversión, en algunos productos la estrategia involucra mayor énfasis en aspectos como la calidad de la fuerza de trabajo, las capacidades organizacionales y la especificidad del mercado (Eden y Monteils 2002). Uno de los ejemplos más claros es el de la manufactura de autopartes, en las plantas que producen conforme a esquemas de coordinación "justo a tiempo", con las ensambladoras de autos o de módulos automotrices. Pero este tipo de factores son válidos para todo el sector exportador y no sólo para las maquiladoras.

\footnotetext{
3 De hecho, estos autores concluyen que la evidencia econométrica a este respecto muestra una influencia negativa (aunque poco significativa) del TLCAN en el crecimiento de las maquiladoras.
} 
Sargent y Matthews $(2003,58)$ mencionan tres aspectos específicos de la regulación de las maquiladoras, directamente afectados por las disposiciones del TLCAN: a) vender la totalidad de su producción en México, a partir del 2001; b) establecer porcentajes de contenido regional obligatorio, para calificar como productos libres de impuestos en Estados Unidos, aunque en ciertos casos los insumos procedentes de otras regiones pueden contabilizarse como regionales si son procesados en México; c) prohibir la importación libre de impuestos para insumos no producidos en los países del TLCAN, que entró en vigor con el artículo 303 en 2001. Durante los primeros años fue posible eludir el pago de impuestos para muchos insumos procedentes de otras regiones.

Uno de los episodios más conflictivos, relacionados con el TLCAN, ocurrió a principios de 2001 y durante los dos años siguientes, cuando entró en vigor la obligación de gravar las importaciones de insumos, según lo previsto por el artículo 303 del tratado. En un intento por mantener la competitividad de las maquiladoras y otras empresas exportadoras, el gobierno estableció los Programas Sectoriales (PROSEC), que otorgan tarifas preferenciales (entre 0 y 5 por ciento) para algunos insumos. Sin embargo, la información sobre las reglas de operación de estos programas fue incompleta, y muchas empresas no pudieron calificar durante los primeros meses de su vigencia. Esto agregó un factor más de incertidumbre a un ambiente de por sí cargado de confusión y pesimismo, debido a la recesión estadounidense y la participación creciente de China en el mercado mundial. Pese a ello, y no obstante las quejas reiteradas de los empresarios por la complejidad de las normas, que obliga a las maquiladoras a cerrar para irse a otros países, no existe evidencia clara de que este factor influya en algo.

En suma, se puede hablar de un "periodo del TLCAN", más que de su efecto directo, en el que las reglas de operación se ajustaron para adecuarse a sus normas nuevas y en el cual son los factores tradicionales los que explican el crecimiento de las maquiladoras, como los ciclos económicos de Estados Unidos y la diferencia de salarios, y por ventajas dinámicas como las necesidades de coordinación cercana según esquemas de producción justo a tiempo.

\section{Aprendizaje tecnológico y capacidades locales}

Un estereotipo muy popular en la literatura más politizada, imperante en los años setenta y ochenta, presenta a las maquiladoras como plantas de ensamble simple, con predominio de trabajo manual no calificado, tecnología rudimentaria y salarios bajos, así como también algunos estudios recientes (De la Garza 2005; De la O y Quintero 2002).

Sin embargo, la transformación del entorno industrial de las maquiladoras se inició hace más de 20 años. A partir de 1980, muchas de las plantas nuevas (sobre todo en las industrias electrónica y de autopartes) introdujeron tecnologías de producción basadas en la microelectrónica, la robótica y de la información. Algunas empezaron a utilizar máquinas de inserción automática de componentes, equipos 
de prueba computarizados, sistemas programables para el traslado de materiales y robots tipo pick and place. Al principio, esta tecnología continúa siendo la excepción, en un contexto caracterizado por un predominio amplio del trabajo manual. Sin embargo, en las industrias electrónica y de autopartes la utilización de tecnologías flexibles y la automatización se difundieron con rapidez (Wilson 1992; Carrillo y Hualde 1998; Lara 1998; Contreras 2000).

La tipología analítica más útil para captar este proceso evolutivo es la de Carrillo y Hualde (1998), quienes identifican tres "generaciones" de empresas: las de primera, dedicadas a actividades de ensamble con predominio de mano de obra, en las de segunda imperan los procesos de manufactura con mucha tecnología y las de tercera, además de manufactura realizan actividades que requieren conocimiento, tales como diseño e investigación y desarrollo. Aunque cada generación surgió en periodos sucesivos, y refleja etapas progresivas de maduración de las capacidades tecnológicas y organizacionales, en realidad la aparición de la generación más avanzada no desplaza a la anterior, por tanto, actualmente existe una configuración industrial heterogénea, en la que coexisten empresas de las tres generaciones en las regiones que albergan a las maquiladoras.

La investigación más reciente sobre capacidades tecnológicas reportó que en 2002, 56 por ciento de las maquiladoras de electrónica y autopartes utilizaba la mejor tecnología disponible en el mercado mundial, 40 por ciento tenía un alto grado de automatización y 68 contaba con sistemas Enterprise Resource Planning (ERP). El mismo estudio menciona que esas empresas, en promedio, tienen 24 máquinas programables y cinco robots por planta (Carrillo 2004).

La utilización de tecnologías avanzadas produjo un cambio en la composición de la fuerza de trabajo, al requerir más trabajadores calificados, técnicos e ingenieros. En 1980, el porcentaje de técnicos era de 9 por ciento, a mediados de 2006 había llegado a 13. Pero en algunas actividades y regiones el empleo de técnicos es mayor, como es el caso de las maquiladoras electrónicas y de autopartes, que emplea a 15 por ciento, y de las ubicadas en Jalisco (17.5) y Chihuahua (15) (INEGI 2006). Asimismo, la expansión constante de la industria requirió contratar gran cantidad de directivos y administradores. De acuerdo con un estudio, durante la década de los noventa tan sólo en Tijuana y Ciudad Juárez se crearon unos 500 nuevos puestos gerenciales cada año, y siete de cada diez de ellos fueron ocupados por mexicanos (Contreras 2000).

Como trayectoria de aprendizaje industrial, han considerado algunos autores a la experiencia acumulada por las regiones donde se han asentado las maquiladoras. De acuerdo con esta interpretación, la acumulación de capacidades tecnológicas termina por convertirse en una ventaja competitiva regional (Ohno y Okamoto 1994). En cuanto a los agentes, la formación de tales capacidades tiene dos fuentes principales: la técnica y profesional de los empleados, basada en el sistema educativo y en los programas de capacitación de las empresas, y la experiencia compartida por esos empleados dentro de las empresas y a través de las redes socio-profesionales locales (Contreras y Kenney 2002). 
Pero estos procesos de aprendizaje no surgen de manera espontánea, sino en el contexto de las presiones competitivas del mercado global. Ninguna firma, ni siquiera las empresas líderes del mercado, puede generar internamente las diversas capacidades exigidas por la competencia global. Por lo tanto, un aspecto decisivo de la competitividad consiste en la habilidad para proveerse de especialistas fuera de la firma; desde una subcontratación simple de la fase de ensamble, hasta procesos sofisticados de diseño (Ernst y Kim 2002). Según esta lógica, las empresas transnacionales con operaciones de maquila en México necesitan transferir capacidades técnicas y gerenciales a sus filiales y proveedores locales, para que sean capaces de cumplir con sus exigentes estándares de calidad y a la vez disminuir sus costos de producción. Una vez que han logrado elevar sus capacidades, los nuevos estándares alcanzados se convierten en un incentivo para delegar conocimientos y procesos más sofisticados en los proveedores locales, incluso la ingeniería, el desarrollo de productos y de procesos.

Aunque muchas transnacionales prefieren centralizar la toma de decisiones y las actividades de investigación y desarrollo (ID) en su propio país, desde el principio de los años noventa tanto las firmas de EE UU como de otros países empezaron a descentralizar algunas de estas actividades. La razón principal es la búsqueda de ventajas estratégicas, tales como la dotación de capital humano (por ejemplo el costo de ingenieros y gerentes), el incremento de las ventas en los mercados locales y el cumplimiento de requisitos comerciales y legales (Eden y Monteils 2002). En el caso de las operaciones de maquila en México, la descentralización de ID es incipiente, en cambio, está muy avanzada la transferencia de la ingeniería del proceso y del producto (Alonso, Carrillo y Contreras 2002), así como de las principales funciones gerenciales (Contreras y Kenney 2002). No obstante su carácter incipiente, la transferencia de actividades de ID también empieza a cobrar relevancia; el estudio de Carrillo (2004) identificó un total de 72 centros de investigación y desarrollo instalados en México por corporaciones transnacionales con operaciones de maquila en el país.

Además del aprendizaje tecnológico, la trayectoria industrial de las regiones con mayor concentración de maquiladoras ha favorecido la acumulación de capacidades institucionales. A lo largo de la última década surgieron diversas instituciones locales denominadas "instituciones puente", por algunos autores, y son "[ ...] organizaciones que actúan como enlace y apoyo de la producción. La función que desempeñan se vincula con la generación de un entorno de confianza y certidumbre al armar redes de colaboración entre diferentes actores facilitando la interacción y el aprendizaje” (Casalet 2004, 60). Los ejemplos más consolidados son el Plan Estratégico de Ciudad Juárez y el Centro de Productividad de la Industria Electrónica de Baja California (ProduCen). En su estudio sobre estos dos casos, Moloman encuentra que estas instituciones han adquirido un papel protagónico en sus respectivos entornos a través de servicios de información, capacitación, financiamiento, organización de eventos y coordinación de actividades, entre otros. Un aspecto relevante de ellas es que 
[... fueron producto de las iniciativas de diversos actores locales para responder a la nueva realidad local y de un real interés en buscar nuevas oportunidades y soluciones para sus regiones. Sus esfuerzos han estado encaminados hacia la coordinación de los organismos públicos y privados tanto en el nivel federal, pero sobre todo en el estatal, lo que ha generado un aprendizaje institucional integral orientado al soporte en el desarrollo industrial (Moloman 2006, 125).

En todo caso, en ausencia de una directriz estratégica, emanada de los organismos gubernamentales encargados de la política industrial, es en este tipo de instituciones, y a partir de la experiencia acumulada por los actores locales, donde parece estarse procesando una visión nueva acerca del potencial y las limitaciones del modelo maquilador, así como de las alternativas para el desarrollo industrial del norte de México, en el marco de la economía global.

\section{Conclusiones y perspectivas}

La declinación de las maquiladoras en 2001 desató en México una polémica intensa acerca del futuro de este modelo de industrialización, que parece tan vulnerable a las fluctuaciones del entorno internacional. El declive es atribuible a factores como el comportamiento cíclico de la economía estadounidense y la competencia creciente de China, Centroamérica y el Caribe; otros factores asociados son la fortaleza del peso mexicano, que debilitó la competitividad de las exportaciones, los cambios en la política fiscal hacia las maquiladoras y la implementación del artículo 303 del TLCAN, en el 2001 (Sargent y Matthews 2003).

De acuerdo con un reporte de la GAO (2003), en los primeros años del siglo XXI México perdió cuota de mercado en 47 de 152 categorías de importaciones estadounidenses, mientras que China ganó participación en 35 de esas 47, que incluyen juguetes, muebles, aparatos electrodomésticos, ciertos equipos de televisión y video, ropa y textiles.

Por otra parte, la incorporación de China a la Organización Mundial de Comercio ha reducido significativamente las tarifas para las importaciones de ese país a Estados Unidos. Ello ha ocasionado que muchas maquiladoras japonesas y coreanas revisen sus estrategias: en lugar de producir los componentes electrónicos en Asia, ensamblarlos en México y vender el producto final en Estados Unidos, algunas empresas ya están trasladando la producción y ensamble a Asia, para atender desde ahí al mercado estadounidense (Sargent y Matthews 2003). En sentido contrario, pero con implicaciones similares para México, a partir de 2003 algunas empresas chinas han establecido operaciones de maquila en México. Hacia fines de 2005 se estimaba que había en el país unas 25 plantas de capital chino, dedicadas a producir teléfonos celulares, electrónica de consumo y confección de ropa (Noticias Financieras, 18 de agosto, 2005). En ambos casos, se trata de muestras de la gran pre- 
sencia de China en el mercado mundial, y de la falta de una estrategia mexicana frente a esta nueva y abrumadora irrupción asiática.

Aunque en una escala menor, algo similar ocurre con la competencia de naciones de Centroamérica y el Caribe. El tratamiento otorgado por Estados Unidos a partir de 2000 a los países de la Iniciativa de la Cuenca del Caribe (ICC) ha erosionado la capacidad de México para competir en el mercado estadounidense de ropa, de manera particular porque los costos laborales de esos países son más bajos que los mexicanos (GAO 2003).

Pese a todos estos factores adversos, la instalación de plantas industriales según la lógica de la "producción compartida" sigue siendo una opción atractiva para las transnacionales. A diferencia del ensamble de componentes o la confección de ropa, hay una amplia gama de productos en los que resulta más difícil que la producción se traslade a China o Centroamérica. Ya sea por los imperativos de coordinación cercana de los sistemas de producción justo a tiempo (la industria de autopartes), por los costos de transporte (televisores de formato grande) o por las restricciones sobre contenido regional (la mayor parte de las plantas asiáticas, que incluye la reciente oleada de maquilas de capital chino), México continúa teniendo ventajas competitivas directamente ligadas a su ubicación geográfica.

Sin embargo, parece evidente que el desarrollo industrial del país no puede mantenerse exclusivamente sobre esas bases. Diversos estudios recientes muestran con claridad que la irrupción de China en el mercado mundial está afectando de manera severa a los países y regiones especializados en manufacturas de exportación que utilizan mucha mano de obra (Blázquez, Rodríguez y Santiso 2006; Dussel 2004). Después de casi 40 años de funcionamiento del programa de maquiladoras, en la actualidad el contenido promedio de insumos nacionales es inferior a 5 por ciento, y a pesar de los avances indudables en el traslado a México de operaciones manufactureras de alta tecnología y de algunas actividades de diseño, investigación y desarrollo, no existe aún una estrategia propia para desarrollar una industria competitiva, basada en procesos de alto valor agregado y conocimiento.

En algunas regiones y en ciertos segmentos industriales se han fomentado capacidades tecnológicas y procesos de aprendizaje local, al trasladarse la ingeniería, gerencia y otras funciones clave a las plantas mexicanas. Además, la trayectoria industrial de estas regiones ha favorecido la creación de algunas instituciones locales capaces de cumplir con funciones de coordinación ejecutiva, y generación de propuestas alternativas de desarrollo regional. Tales casos constituyen un claro ejemplo del potencial de creación de capacidades locales, con base en la experiencia acumulada por las maquiladoras, pero también muestran la inexistencia de una política industrial capaz de articular estos esfuerzos locales con una estrategia global de crecimiento económico.

En el pasado, los "tigres asiáticos" crearon sus industrias locales aprovechando la presencia de maquiladoras, mostrando que una estrategia de desarrollo económico y tecnológico puede originarse en dichas industrias, siempre y cuando vaya acompañada de normatividad que incluya estrategias específicas, para generar encadenamientos productivos locales y transferencia tecnológica. Tal fue el caso de 
Corea del Sur, donde las maquiladoras sirvieron como base para crear su propia industria automotriz y electrónica; de Taiwán, con la electrónica mundialmente competitiva; de Malasia, con la textil y eléctrónica propia y de Singapur, con una industria electrónica pujante, que de paso se consolidó como una de las economías más competitivas del mundo (Lam 1992; Kuo 1995; Kenney y Lowe 1999; McKendrick, Doner y Haggard 2000; Rasiah 1995). Más tarde, otras economías emergentes, como la India e Irlanda, levantaron con éxito un segmento empresarial de "clase mundial", en las industrias nuevas ligadas a las tecnologías de la información. En la actualidad, China no sólo atrae la mayor parte de las inversiones para industrias que requieren mucha mano de obra, sino además despliega una capacidad sorprendente de aprendizaje tecnológico y de creación de empresas locales nuevas (Arvanitis y Wei 2003).

México carece de una política industrial coherente y dinámica, capaz de estimular los segmentos de alto valor agregado y conocimiento. La crisis de 2000 a 2003 fue probablemente un anticipo del desastre social y económico que podría ocurrir si México continúa basando sus industrias exportadoras en segmentos de bajo valor agregado en un entorno global de presiones feroces para la reducción de costos de producción. Parte de la respuesta está en las iniciativas y proyectos de los actores nuevos e instituciones locales, depositarios de una larga experiencia en estos mercados complejos y demandantes.

\section{Bibliografía}

Alonso, Jorge, Jorge Carrillo y Óscar Contreras. 2002. Aprendizaje tecnológico en las maquiladoras del norte México. Frontera Norte XIV (27): 43-82.

Arvanitis, Rigas y Zhao Wei. 2003. La industrialización del sur de China: aprendizaje y limitaciones de un modelo exitoso. Espacios XxIv (3): 67-93.

Blázquez-Lidoy, Jorge, Javier Rodríguez y Javier Santiso. 2006. Angel or Devil? Chinesse Trade Impact on Latin American Emerging Markets. Working paper no. 252. París: Organization for Economic Co-operation and Development (OECD) Development Centre.

Capdeville, Mario. 2005. Procesos de producción global: ‘alternativa para el desarrollo mexicano? Comercio Exterior 55 (7): 561-573.

Carrillo, Jorge. 2004. Principales estadísticas de la industria maquiladora. Encuesta sobre aprendizaje tecnológico y escalamiento industrial. Tijuana: El Colegio de la Frontera Norte.

Carrillo, Jorge y Alfredo Hualde. 1998. Third Generation Maquiladora? The DelphiGeneral Motors Case. Journal of Borderland Studies XXIII (1): 79-97. 
Carrillo, Jorge. 1989. Transformaciones en la industria maquiladora de exportación. En Las maquiladoras; ajuste estructural y desarrollo regional, compilado por Bernardo González-Aréchiga y Rocío Barajas, 37-54. Tijuana: El Colegio de la Frontera Norte-Fundación Friedrich Ebert.

Casalet, Mónica. 2004. Construcción institucional del mercado en la economía del conocimiento. Economía UNAM (2): 52-63.

Centro de Estudios de las Finanzas Públicas de la Cámara de Diputados. 2003. Consideraciones generales sobre el régimen fiscal aplicable a las maquiladoras para 2003. Documento. México.

Comisión Económica para América Latina y el Caribe (CEPAL). 1996. México: La industria maquiladora. Documento. Santiago de Chile.

Contreras, Óscar. 2000. Empresas globales, actores locales. Producción flexible y aprendizaje industrial en las maquiladoras. México: El Colegio de México.

Contreras, Óscar y Alfredo Hualde. 2004. El aprendizaje y sus agentes. Los portadores del conocimiento en las maquiladoras del norte de México. Estudios Sociológicos XXII (64): 79-121.

Contreras, Óscar y Martin Kenney. 2002. Global Industries and Local Agents: Becoming a World Class Manager in the Mexico-UsA Border Region. En Communities Across Borders, compilado por Paul Kennedy y Victor Roudometof, 129-142. London and New York: Routledge.

De la Garza, Enrique (coordinador). 2005. Modelos de producción en la maquiladora de exportación. México: Universidad Autónoma Metropolitana (UAM) Iztapalapa-Plaza y Valdés.

De la O, María Eugenia y Cirila Quintero (coordinadoras). 2002. Globalización, trabajo y maquilas: las nuevas y viejas fronteras de México. México: Plaza y Valdés.

Diario Oficial de la Federación. 1998. Decreto para el fomento y operación de la industria maquiladora de exportación. México. 1 de junio.

Dussel, Enrique. 2004. Los efectos económicos de China para México y Centroamérica. México: CEPAL.

Eden, Lorraine y Antonie Monteils. 2002. Regional Integration: NAFTA and the Reconfiguration of North American Industry. En Regions, Globalization and the Knowledge-Based Economy, editado por John H. Dunning, 170-219. Oxford: Oxford University Press. 
Ernst, Dieter y Linsu Kim. 2002. Global Production Networks, Knowledge Diffusion, and Local Capability Formation. Research Policy 31 (8-9): 1417-1429.

General Accounting Office (GAO). 2003. Mexico's Maquiladora Decline Affects U.S.-Mexico Border Communities and Trade; Recovery Depends in Part on Mexico's Actions. Documento. Washington.

Gobierno de los Estados Unidos Mexicanos. Presidencia de la república. 2006. Sexto informe de gobierno deVicente Fox Quesada. México.

Gruben, William y Sherry L Kiser. 2001. NAFTA and Maquiladoras. Is the Growth Connected? Border Economy 7: 22-24.

Instituto Nacional de Estadística, Geografía e Informática (INEGI). 2006. Estadística de la industria maquiladora de exportación, Banco de Información Económica. http://dgcnesyp.inegi.gob.mx/cgi-win/bdieintsi.exe (3 de octubre de 2006).

Kenney, Martin y Nichola Lowe. 1999. Foreign Investment and the Global Geography of Production: Why the Mexican Consumer Electronics Industries Failed. World Development 27 (8): 1427-1443.

Kuo, C. 1995. Global Competitiveness and Industrial Growth in Taiwan and the Philippines. Pittsburgh: University of Pittsburgh Press.

Lam, D. K. 1992. Explaining Economic Development: A Case Study of State Polices Towards the Computer and Electronics Industry in Taiwan. Ph. D. dissertation, Carleton University.

Lara Rivero, Arturo. 1998. Aprendizaje tecnológico y mercado de trabajo en las maquiladoras japonesas. México: Miguel Ángel Porrúa-UAm Xochimilco.

McKendrick, David, Richard F. Doner y Stephan Haggard. 2000. From SiliconValley to Singapore: Location and Competitive Advantage in the Hard Disk Drive Industry. Stanford: Stanford University Press.

Moloman, Elena. 2006. Modelo de exportación, desarrollo de capacidades y surgimiento de un nuevo tipo de instituciones. Comparación de Tijuana y Ciudad Juárez.Tesis de maestría en Desarrollo Regional, El Colegio de la Frontera Norte.

Noticias Financieras. 2006. Remarcan existencia de hasta 25 maquiladoras chinas en México. 18 de agosto.

Ohno, Koichi y Yumiko Okamoto. 1994. Regional Integration and Foreign Direct Investment: Implications for Developing Countries. Tokyo: Institute of Developing Economies. 
Rasiah, R. 1995. Foreign Capital and Industrialization in Malaysia. New York: St. Martin’s Press.

Sargent, John y Linda Matthews. 2003. Boom and Bust: Is it the End of Mexico's Maquiladoras? Business Horizons 46 (2): 57-64.

Sargent, John y Linda Matthews. 2003. What Happens when Relative Costs Increase in Export Processing Zones? Technology, Regional Production Networks and Mexico's Maquiladoras. Working paper. Edinburg: Center of Border Economic Studies-The University of Texas-Pan American.

Vargas Leyva, María Ruth. 2003. Industria maquiladora de exportación. ¿Hacia dónde va el empleo? Papeles de Población 37: 243-269.

Villarreal, M. Ángeles. 2003. Industry Trade Effects related to NAFTA. Washington: Congressional Research Service.

Wilson, Patricia. 1992. Exports and Local Development: Mexico's New Maquiladoras. Austin: University of Texas Press. 
